\title{
Análise imuno-histoquímica das sintases do óxido nítrico em adenocarcinomas gástricos
}

\section{Immunohistochemical expression of nitric oxide synthases in gastric adenocarcinomas}

Maria Dirlei F. S. Begnami'; Isabela Werneck da Cunha'; André Montagnini²; Sueli Nonogaki³; Fernando Soares ${ }^{4}$

unitermos
Câncer gástrico
Óxido nítrico

Sintases do óxido nítrico

\section{resumo}

Introdução: O óxido nítrico (NO) é uma molécula mediadora de diversas funções, tais como vasodilatação, neurotransmissão e metabolismo do ferro. Os efeitos do NO na biologia tumoral são ambíguos e complexos. A atividade das sintases do óxido nítrico (NOS) tem sido demonstrada em diversos tumores humanos. Objetivo: Pesquisar a expressão das isoformas das NOS em carcinomas gástricos e correlacionar estes achados com características demográficas e histopatológicas destes tumores. Material e métodos: $\mathrm{O}$ estudo imuno-histoquímico das NOS (NOS-1, NOS-2 e NOS-3) foi realizado em 128 casos de carcinomas gástricos classificados de acordo com o sistema de Lauren. Resultados: A positividade para NOS-1 foi detectada em 92/128 (70\%) dos casos, para NOS-2 em 36/128 (30\%) e para NOS-3 em 54/128 (42\%) dos casos. Na análise estatística observou-se correlação com o tipo intestinal e expressão de NOS-3, e tumores avançados mostraram maior expressão de NOS-2. Conclusão: Os carcinomas gástricos mostram expressão das três isoformas de NOS, sendo as NOS constitutivas presentes em maior número de casos. A freqüente expressão de NOS induzida nos carcinomas gástricos avançados sugere uma participação de NOS na progressão e na disseminação tumoral na mucosa gástrica. abstract

Introduction: Nitric oxide is an important bioactive and signaling molecule that mediates a diverse array of actions such as vasodilatation, neurotransmission, and iron metabolism. Also, it can act as a carcinogen. Recent studies have examined the expression and activity of the NOS isoforms in several human cancers. Objectives: To investigate the expression of nitric oxide synthases in gastric carcinomas and correlate the results. Material and methods: The immunohistochemistry expression of constitutive and inducible nitric oxide synthases (NOS-1, NOS-2 e NOS-3) were evaluated in 128 cases of gastric cancer classified according to Lauren system. Results: The rate of expression of NOS-1 was 92 (70\%) of the 12 cases, NOS-2 was 36 (30\%) and NOS-3 was 54 (42\%) of the cases. The expression of NOS-3 was associated with the intestinal type of carcinoma and deeply invasive tumors showed high rate of expression of NOS-2. Conclusion: There is high expression of all the isoforms of nitric oxide synthases in gastric cancer; the constitutive isoforms show higher expression than the inducible forms. The high expression of the inducible form in deeply invasive tumors is related with tumoral progression and dissemination in the gastric mucosa. key words

Gastric cancer

Nitric oxide

Nitric oxide synthases 


\section{Introdução}

O câncer de estômago é uma das neoplasias mais freqüentes em nosso meio com alta taxa de incidência e mortalidade ${ }^{(13)}$. Mais de $90 \%$ dos casos são constituídos por adenocarcinomas, que podem ser divididos, segundo Lauren, em dois subtipos histológicos principais: o intestinal e o difuso(11). A etiopatogenia do câncer gástrico é multifatorial, sendo que as informações sobre o mecanismo de carcinogênese gástrica são motivo de amplo debate na literatura.

O óxido nítrico (NO, do inglês nitric oxide) é uma molécula importante como sinalizadora de diversas funções tais como vasodilatação, neurotransmissão, regulação da apoptose, carcinogênese e metabolismo do ferro ${ }^{(2,14)}$. A ação está na dependência da concentração. O NO é um gás incolor, altamente lipossolúvel, difundindo-se facilmente entre as células, e produzido a partir de uma reação enzimática, na qual o substrato L-arginina é convertido em L-citrulina e NO. Sabe-se que a reação de conversão é catalisada por uma família de enzimas, conhecidas como sintases do óxido nítrico (NOS, do inglês nitric oxide synthases). As NOS apresentam três isoformas, que são agrupadas em dois grupos: as constitutivas (cNOS) e as induzidas (iNOS). As NOS-1 (ou NOS neuronal) e as NOS-3 (ou NOS endotelial) são constitutivas, enquanto que as NOS-2 (ou NOS macrofágica) são induzidas. A atividade das cNOS é dependente de cálcio e calmodulina. As NOS-2 não são expressas fisiologicamente, mas apenas após a ativação por citocinas inflamatórias e lipopolissacarídeos bacterianos. Uma das características diferenciais das NOS-2 é serem cálcio e calmodulina-independentes ${ }^{(22)}$. A regulação das NOS-2 permite que altos níveis de NO (nmoles) possam ser produzidos e mantidos, ao contrário do que ocorre nas NOS-1 e nas NOS-3, que produzem baixos níveis de NO (pmoles) ${ }^{(19)}$. Tanto a quantidade de NO produzido quanto a duração desta produção são fundamentais para definir se o efeito produzido pelas NOS será benéfico ou prejudicial à saúde. Em geral, as ações tóxicas do NO estão relacionadas com o produto das NOS-2 e não com os produtos das NOS-1 e $3^{(15)}$.

Na mucosa gástrica normal, o NO inibe a secreção gástrica, estimula a produção de muco e de secreções bicarbonadas, eleva o fluxo sanguíneo na mucosa e favorece o processo de cicatrização de úlceras ${ }^{(3,9)}$. Tanto as formas constitutivas (cNOS) como as induzidas (iNOS) têm sido encontradas na mucosa gástrica normal(4).
Na biologia tumoral, os efeitos do NO são complexos e dualísticos, ora favorecendo a gênese, o crescimento, a angiogênese e a invasão tumoral, ora com ações antitumorais, pela citotoxicidade e pela indução da apoptose ${ }^{(1,10,15)}$. Estudos recentes têm demonstrado a expressão e a atividade das três isoformas das sintases do óxido nítrico (NOS) em tumores humanos de diversos sítios e histogênese, incluindo o estômago. Em estudo de peças de adenocarcinomas gástricos, Rajnakova et al. (1997) mostraram que as células neoplásicas expressam menores quantidades de NOS do que aquelas da mucosa normal, enquanto que tumores avançados tinham maior expressão de NOS-2 do que aqueles mais precoces ${ }^{(17)}$. A expressão e a atividade de NOS-2 estão aumentadas em tumores gástricos avançados e nas metástases hepáticas ou pulmonares ${ }^{(7)}$.

Há poucos estudos na literatura em relação à expressão das NOS constitutivas nos carcinomas gástricos. Aparentemente, a expressão das NOS constitutivas está relacionada com o estádio do tumor, sendo que carcinomas gástricos avançados (T4) expressam menores quantidades de NOS-1 e NOS-3 do que aqueles em estádio T2 ${ }^{(17)}$.

Outro aspecto é que, aparentemente, o NO tem participação na carcinogênese gástrica. A expressão imunohistoquímica de NOS-2 e nitrotirosina foi significativamente maior em biópsias com a presença de $H$. pylori do que naquelas $H$. pylori-negativas e também significativamente maior na mucosa de pacientes que desenvolveram carcinomas gástricos até dois anos depois da biópsia(12). Resultado semelhante em mucosas gástricas foi obtido por Hahm et al. (1998): mucosas de pacientes com infecção pelo H. pylori apresentaram maiores quantidades de NOS-2 e maior índice apoptótico ${ }^{(8)}$. Esses resultados abrem uma interessante associação entre a nitrificação produzida pela infecção pelo H. pylori via NOS, dano oxidativo do DNA, apoptose e a carcinogênese gástrica.

O objetivo deste trabalho é determinar através de técnica de imuno-histoquímica a expressão das NOS, constitutivas e induzidas, em carcinomas gástricos, e correlacionar esta expressão com as variáveis demográficas e morfológicas dos tumores e presença de infecção pelo $H$. pylori.

\section{Material e método}

Foram selecionados 128 casos de carcinomas gástricos submetidos à gastrectomia total ou parcial no período de 1995 a 1998, obtidos nos arquivos da anatomia patológica do Centro de Tratamento e Pesquisa do Hospital do Cân- 
cer A. C. Camargo. Foram resgatadas todas as respectivas lâminas e blocos de parafina. Blocos representativos do tumor foram selecionados e novos cortes foram realizados. O primeiro corte obtido de cada bloco foi corado pela técnica tradicional de hematoxilina e eosina (HE). A revisão da lâmina permitiu o diagnóstico histológico da neoplasia, separando os tumores nos tipos intestinal e difuso de acordo com a classificação de Lauren ${ }^{(11)}$. Cortes corados pelo Giemsa foram realizados para a pesquisa de $H$. pylori em mucosa não-tumoral distante da neoplasia.

Os prontuários médicos dos casos selecionados foram revistos, sendo resgatadas as informações demográficas como idade, gênero e raça.

As reações imuno-histoquímicas foram realizadas nos cortes com neoplasia, pela técnica de complexo estreptavidina-biotina-peroxidase (StreptABC, DAKO ${ }^{\circledR}$ ). Resumidamente, as lâminas foram previamente revestidas por solução de silano (APTS - Sigma ${ }^{\circledR}$ A3648) diluído a 4\% em acetona. Os cortes obtidos foram de $3 \mu \mathrm{m}$ de espessura após microtomia mecânica. Os cortes foram desparafinizados e preparados por passagens sucessivas por xilol e etanol e submetidos à recuperação antigênica pelo calor com irradiação por panela de pressão (Eterna, Nigro) utilizando-se tampão citrato $10 \mathrm{mM} \mathrm{pH} 6.0$ por 15 minutos. O bloqueio da peroxidase endógena foi realizado com solução de peróxido de hidrogênio a 3\%, seguida da incubação com os anticorpos primários NOS-1 (nNOS, 1:200, Transduction Laboratories, USA), NOS-2 (iNOS, 1:40, Transduction Laboratories, USA) e NOS-3 (eNOS, 1:50, Transduction Laboratories, USA). As reações foram reveladas com solução de diaminobenzidina $\left(\mathrm{DAB}\right.$, Sigma $^{\circledR}$ ) a $60 \mathrm{mg} \%$ e contracoradas com hematoxilina de Harris (Merck ${ }^{\circledR}$ ). Para cada reação utilizou-se, como controle positivo, tecido sabidamente positivo para o anticorpo testado. Dois controles negativos foram também utilizados, sendo o primeiro deles pelo não-uso do anticorpo primário e o segundo, através da retirada do anticorpo secundário durante os passos da reação.

Os casos foram considerados positivos para NOS quando a coloração marrom era visível no citoplasma da célula tumoral em intensidade pelo menos moderada e em mais do que $10 \%$ das células. Não foi considerada a expressão em células não-neoplásicas.

A análise estatística foi realizada utilizando-se o programa de computador SPSS 10.0 (SPSS Inc., Chicago,IL, USA). Para a análise da associação entre dois grupos foi utilizado o teste qui-quadrado ou o teste exato de Fisher. Foram considerados significantes os valores de alfa $<0,05$.

\section{Resultados}

A mediana da idade dos pacientes foi de 63 anos, e a média foi de 61 anos (25-84 anos). Os achados demográficos e morfológicos estão descritos na Tabela 1. A maioria dos pacientes (69\%) era do sexo masculino e de etnia branca (85\%). Em relação ao tipo histológico, 72 casos (57\%) eram do tipo intestinal, e 56 (43\%), do tipo difuso. As lesões eram predominantemente do tipo infiltrativas, com infiltração da serosa/subserosa em 92/128 casos (72\%). Em 78 casos (60\%) foram identificadas metástases em linfonodos. A presença de formas bacterianas compatíveis com H. pylori pelo método de Giemsa foi detectada em 40 casos (32\%).

A expressão de NOS-1 foi detectada em 92 casos (70\%); a de NOS-2, em 36 casos (30\%) e a de NOS-3, em $54(42 \%)$ dos 128 casos (Figura 1). Quando analisados os casos individualmente, 98 casos (83\%) expressaram pelo menos uma das isoformas de NOS, sendo que em 29 casos (23\%) observou-se expressão concomitante de NOS-1 e NOS-2; em 22 casos (17\%), de NOS-2 e NOS-3; e em 44 casos (37\%), expressão de NOS-1 e NOS-3. Exemplos da expressão de NOS-1, NOS-2 e NOS-3 podem ser vistos na Figura 2. A expressão das três isoformas das NOS não mostrou associação com idade, gênero, etnia, localização do tumor, metástases em linfonodos e infecção pelo $H$. pylori (Tabela 2).

\section{Tabela 1 gástricos}

\begin{tabular}{lcc}
\hline Variável & Categoria & $\begin{array}{c}\text { Número } \\
\text { de casos }\end{array}$ \\
Sexo & $\begin{array}{c}\text { Masculino } \\
\text { Feminino }\end{array}$ & $\begin{array}{c}80(70 \%) \\
\text { Raça }\end{array}$ \\
& Branca & $108(85 \%)$ \\
Tipo histológico & Não-branca & $20(15 \%)$ \\
& Intestinal & $72(57 \%)$ \\
Infecção pelo H. pylori & Difuso & $56(43 \%)$ \\
& Presente & $40(32 \%)$ \\
Metástases linfonodais & Ausente & $88(68 \%)$ \\
& Presente & $78(60 \%)$ \\
Total & Ausente & $50(40 \%)$ \\
& & $128(100 \%)$ \\
\hline
\end{tabular}




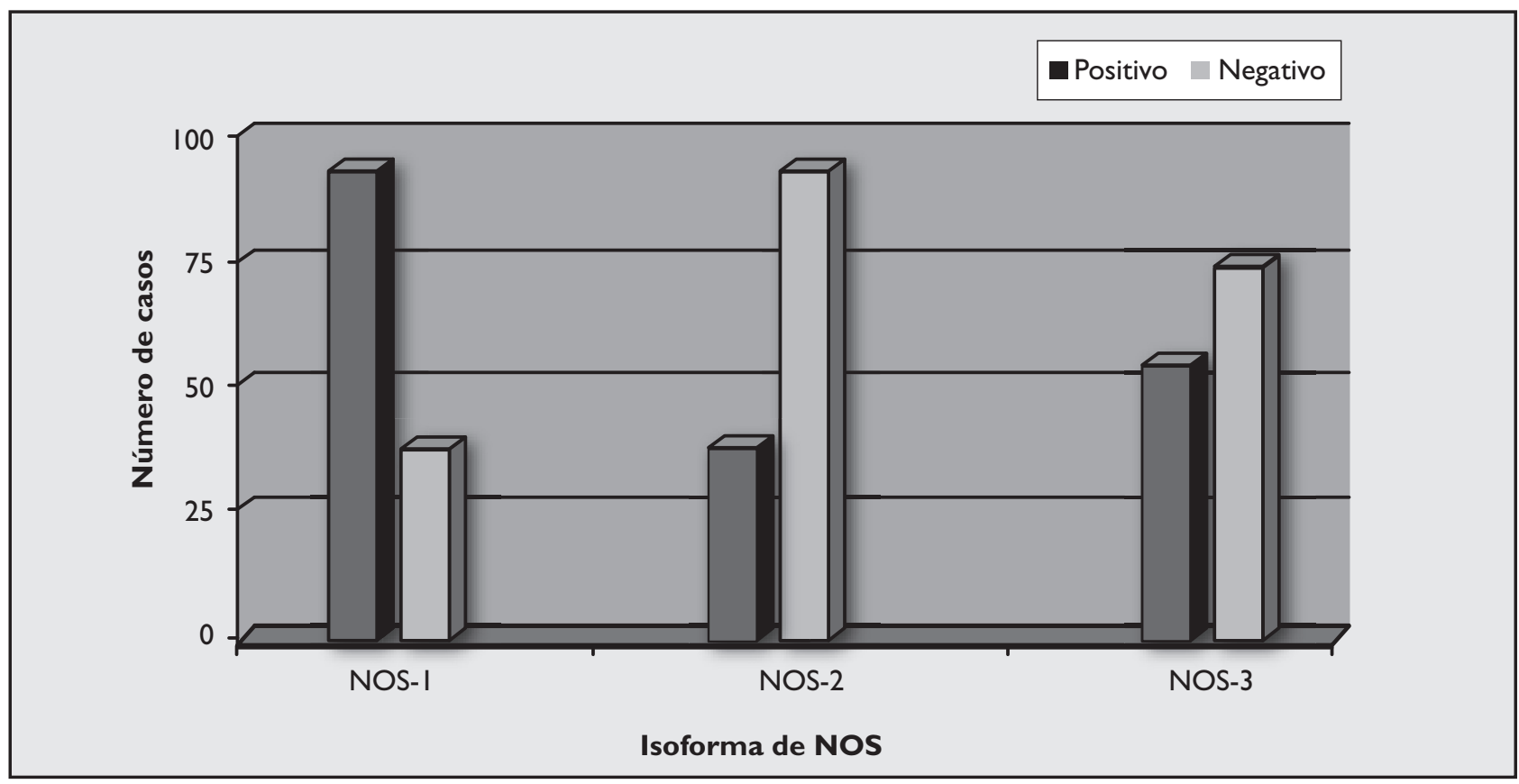

Figura 1 - Expressão imuno-histoquímica das sintases do óxido nítrico (NOS) em carcinomas gástricos

O resultado comparativo entre expressão de NOS-1, NOS-2 e NOS-3 e nível de infiltração do tumor e tipo histológico está demonstrado na Figura 1. NOS-1 foi mais freqüentemente expresso em casos do tipo intestinal ( $52 \%$ dos casos) do que no tipo difuso ( $48 \%$ dos casos) e essa diferença não foi significativa. A expressão de NOS-2 também não mostrou correlação com o subtipo histológico $(p=0,16)$.

A expressão de NOS-3 foi observada em 54 (42\%) dos 128 casos estudados. A positividade de NOS-3 foi mais observada em carcinomas do tipo difuso do que nos intestinais, sendo positiva em $55 \%$ dos casos dos carcinomas difusos e em $45 \%$ dos casos do tipo intestinal $(p=0,04)$.

A expressão de NOS-2 nos carcinomas gástricos foi observada principalmente nos carcinomas com infiltração mais profunda, sendo que dos 36 casos positivos para NOS2, 31 casos (85\%) infiltravam serosa/subserosa, enquanto que somente cinco casos (15\%) infiltravam a muscular/ submucosa. Esse resultado mostrou-se estatisticamente significativo $(p=0,01)$.

\section{Discussão}

O presente estudo demonstrou a relação entre a expressão das sintases do oxido nítrico e algumas características clinicopatológicas dos carcinomas gástricos, como a correlação da expressão de NOS-3 e o tipo intestinal, e entre a expressão de NOS-2 e o nível de infiltração da parede gástrica. No nosso estudo, os carcinomas gástricos expressam alguma das isoformas de NOS em cerca de $80 \%$ dos casos. A isoforma mais freqüentemente expressa é a NOS-1 (70\% dos casos), seguida de NOS-3 (42\% dos casos) e finalmente NOS-2 (30\% dos casos). Poucos trabalhos na literatura realizaram o estudo com as três isoformas de NOS. Em estudo através da detecção de mRNA, Doi et al. (1999) demonstraram que praticamente todos os casos de adenocarcinomas gástricos expressam NOS-3, em quantidades similares à mucosa normal ${ }^{(5)}$. Desta forma, nosso trabalho mostra que as células neoplásicas dos adenocarcinomas gástricos têm o aparato enzimático para a produção de NO, preferencialmente as NOS constitucionais.

O NO é produzido a partir do substrato L-arginina que é convertido através das enzimas em L-citrulina e NO. As NOS constitutivas são responsáveis pela produção de pequenas quantidades de NO, enquanto que a NOS induzida é responsável por quantidades maiores e ação mais duradoura ${ }^{(19)}$. Os resultados do presente estudo mostram que, apesar de mais de $80 \%$ dos tumores expressarem alguma forma de NOS, somente $30 \%$ expressam a NOS2. A revisão da literatura mostra resultados similares ${ }^{(18)}$ ou discretamente mais elevados, em torno de $44 \%-50 \%$ dos $\operatorname{casos}^{(6,20,21)}$. Maiores quantidades e freqüência de expressão de NOS induzida têm sido associadas a doenças em estádios mais avançados ${ }^{(5,18)}$. O significado dessa diferença em relação à produção de NO necessita ainda ser avaliado 


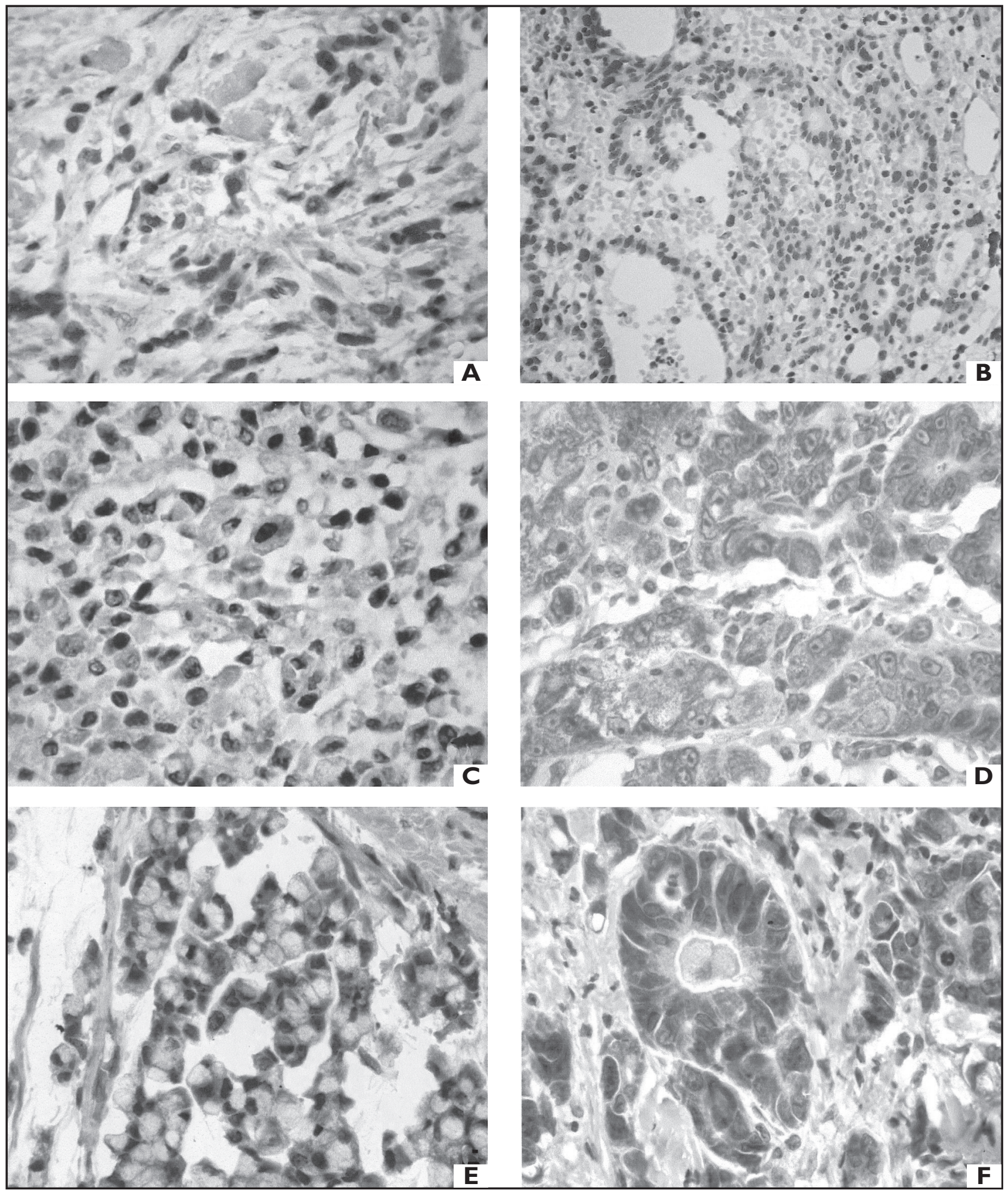

Figura 2 - Imunomarcação para NOS em adenocarcinomas gástricos do tipo difuso ( $A, C$ e E) e intestinal (B, D e F). A e B: exemplos de reação para NOS-1; C e D: casos positivos para NOS-2; E e F: imunorreatividade para NOS-3

pela quantificação sérica e tecidual. O nosso estudo demonstra a correlação da expressão das sintases do óxido nítrico com os tumores mais avançados que infiltravam a serosa e/ou subserosa, porém a correlação com metástases em linfonodos não mostrou diferença estatisticamente significativa. A hipóxia, presente nos tumores avançados, e os mecanismos de angiogênese ativados nesses tumores podem ser os responsáveis pelo aumento de expressão de 
Expressão de NOS-1, NOS-2 e NOS-3 nos tumores gástricos em relação às principais

Tabela 2 características demográficas e histopatológicas

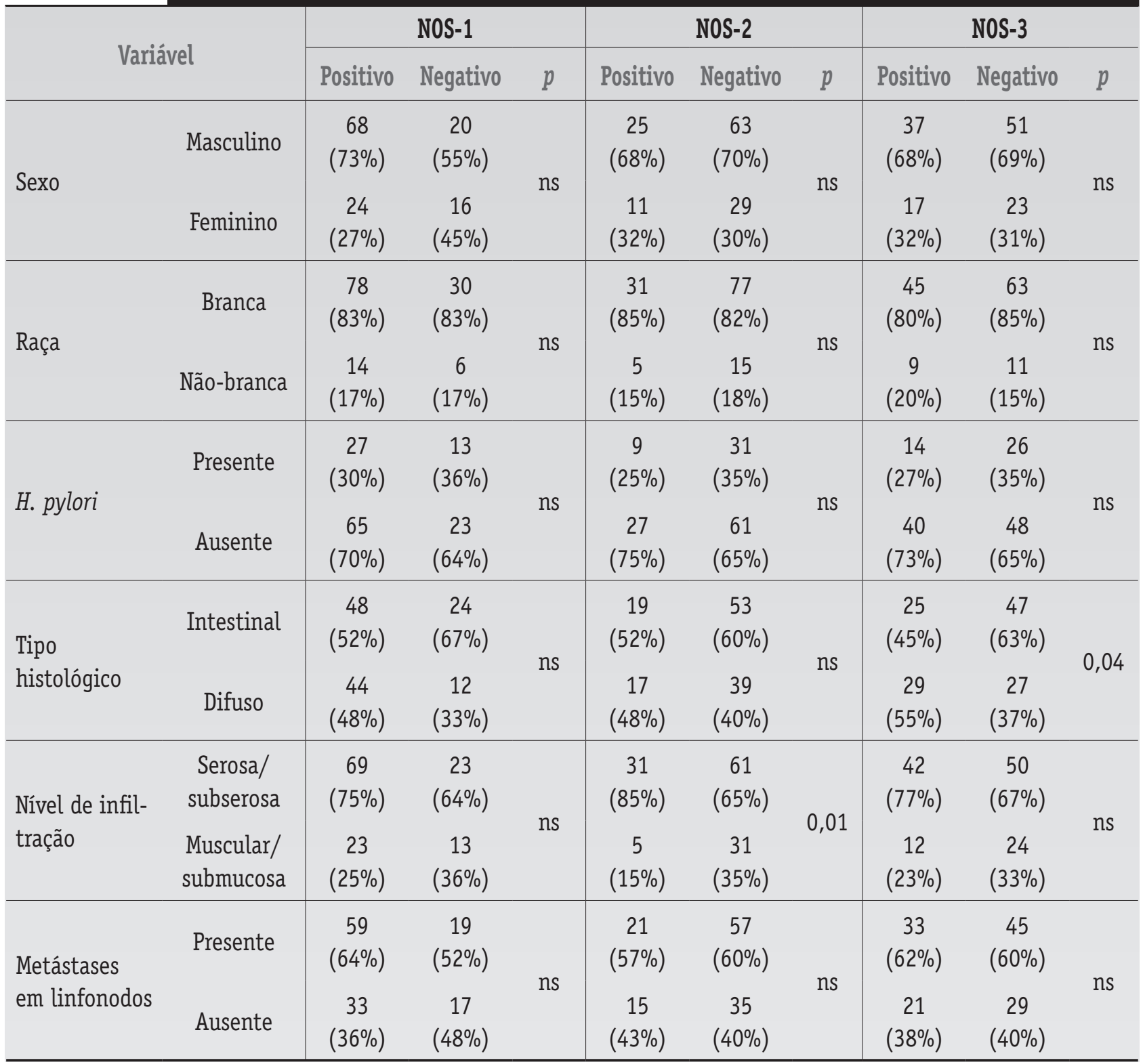

NOS-1 = sintases do óxido nítrico 1; NOS-2 = s

$n s=$ não-significativo.

NOS observado nesses casos. Outro aspecto a ser considerado é que os carcinomas avançados podem conter lipopolissacarídeos produzidos por bactérias gram-negativas e citocinas originadas de linfócitos que permeiam o tumor e que estimulam a produção de NOS. Sendo assim, a produção de NO elevada pode favorecer o crescimento e a disseminação tumoral através do estímulo angiogênico, do aumento da permeabilidade vascular e da supressão da resposta imune.

Não observamos correlações entre as expressões de NOS e as demais variáveis clínicas, como idade, sexo e lo- calização do tumor. A associação com o subtipo histológico foi vista somente em relação ao NOS-3 e o tipo intestinal, não sendo vista nas demais isoformas. Esse achado é diferente do descrito por Son et al. (2001), em que não foram encontradas diferenças estatisticamente significativas entre os subtipos histológicos e a expressão de NOS(20).

No nosso estudo, semelhante ao descrito por Son et al. (2001), também não encontramos correlação positiva entre H. pylori e expressão de $\operatorname{NOS}^{(20)}$. Ao contrário, alguns trabalhos têm demonstrado uma expressão aumentada de iNOS nos tumores H. pylori-positivos ${ }^{(12,16)}$. Essa diferença de 
resultados pode ser justificada pela diferença de métodos utilizados tanto na pesquisa de $H$. pylori (PCR $\times$ Giemsa) quanto na expressão de NOS (PCR x imuno-histoquímica), o que nos permite afirmar que muitos casos de infecção pelo $H$. pylori não são adequadamente detectados pelos métodos usuais de pesquisa.

Em conclusão, o nosso estudo demonstrou que carcinomas gástricos expressam as três isoformas de NOS, sendo NOS-1 a isoforma mais expressa (70\%). Os carcinomas que expressam NOS-3 são freqüentemente do tipo intestinal e há uma baixa expressão de NOS-2, sem correlação com infecção pelo H. pylori. Os carcinomas gástricos mais avançados mostram aumento de expressão de NOS-2. Estes achados, portanto, favorecem a hipótese de que a participação do oxido nítrico na progressão tumoral e a regulação da produção de NO por agentes, como inibidores de NOS, pode representar um novo alvo terapêutico, principalmente nos carcinomas avançados.

\section{Referências}

I. AMBS, S. et al. p53 and vascular endothelial growth factor regulate tumor growth of NOS-2-expressing human carcinoma cells. Nat Med, v. 4, p. I37|-6, 1998

2. BREDT, D. S.; SNYDER, S. H. Nitric oxide: a novel neuronal messenger. Neuron, v. 8, p. 3-I I, 1992.

3. BROZOZOWSKI, T. et al. Role of L-arginine, a substrate for nitric oxide-synthase, in gastroprotection and ulcer healing J Gastroenterol, v. 32, p. 442-52, 1997.

4. BROWN,J. F. et al. Lipopolysaccharide induces $\mathrm{Ca}^{2+}$ independent nitric oxide synthase activity in rat mucosal cells. Eur J Pharmacol, v. 292, p. I I-4, 1994.

5. DOI, C. et al. Expression of nitric oxide synthase in gastric cancer. Cancer Lett, v. |44, p. 161-7, 1999

6. FENG, C. W. et al. Expression of p53, inducible nitric oxide synthase and vascular endothelial growth factor in gastric precancerous and cancerous lesions: correlation with clinical features. BMC Cancer, v. 2, n. 8, 2002.

7. GOTO, T. et al. Enhanced expression of inducible nitric oxide synthase and nitrotyrosine in gastric mucosa of gastric cancer patients. Clin Cancer Res, v. 5, p. I4 I I-5, 1999.

8. HAHM, K. B. et al. Helicobacter pylori, oxidative DNA damage, gastric carcinogenesis, and reversibility by rebamipide. Diag Dis Sci, v. 43, 72S-77S, 1998

9. KATO, S. et al. Role of nitric oxide in regulation of gastric acid secretion in rats: effects of $\mathrm{NO}$ donors and synthase inhibitor. BrJ Pharmacol, v. 123, p. 839-46, 1998.

10. LALA, P. K.; ORUCEVIC, A. Role of nitric oxide in tumor progression: lessons from experimental tumors. Cancer Metastasis Rev, v. 17, p. 91-106, 1998.

I I. Lauren P.The two histological main types of gastric carcinoma. Diffuse and so-called intestinal type carcinoma: an attempt at histoclinical classification. Acta Pathol Microbiol Scand, v. 64, p. 3I-49, 1965.

12. MANNICK, E. E. et al. Inducible nitric oxide synthase, nitrotyrosine, and apoptosis in Helicobacter pylori gastritis: effect of antibiotics and antioxidants. Cancer Res, v. 56, p. 3238-43, 1996.

1 3. MINISTÉRIO DA SAÚDE. Secretaria Nacional de Assistência à Saúde. Instituto Nacional do Câncer. Estimativa da incidência e mortalidade por câncer no Brasil. Rio de Janeiro: INCA; 2002
14. MONCADA, S.; PALMER, R. M.; HIGGS, E. A. Nitric oxide: physiology, pathophysiology, and pharmacology. Pharmacol Rev, v. 43, p. 109-42, 1991.

15. OHSHIMA, H.; BARTSCH, H. Chronic infections and inflammatory processes as cancer risk factors: possible role of nitric oxide in carcinogenesis. Mutat Res, v. 305, p. 253-64, 1994

16. PIGNATELLI, B. et al. Inducible nitric oxide synthase, antioxidative enzymes and Helicobacter pylori infection in gastritis and gastric precancerous lesions in humans. Eur J Cancer Prev, v. 7, p. 439-47, 1998.

17. RAJNAKOVA, A. et al. Expression of differential nitric oxide synthase isoforms in human normal gastric mucosa and gastric cancer tissue. Carcinogenesis, v. 18, p. I84|-5, 1997.

18. RAJNAKOVA, A. et al. Expression of nitric oxide synthase, cyclooxygenase, and p53 in different stages of human gastric cancer. Cancer Lett, v. 172, p. 177-85, 2001.

19. SCHMIDT, H. H. et al. Ca2+/calmodulin-regulated nitric oxide synthases. Cell Calcium, v. I3, p. 427-34, 1992.

20. SON, H. J. et al. Interaction between cyclooxygenase-2 and inducible nitric oxide synthase in gastric cancer. J Clin Gastroenterol, v. 33, p. 383-8, 2001

2।. SONG, Z. J.; GONG, P.; WU, Y. E. Relationship between the expression of iNOS, VEGF, tumor angiogenesis and gastric cancer. World J Gastroenterol, v. 8, p. 591-5, 2002.

22. WOLF, G. Nitric oxide and nitric oxide synthase: biology, pathology, localization. Histol Histopathol, v. 12, p. 25।-6I, 1997.

\section{Endereço para correspondência}

Maria Dirlei F. S. Begnam

Departamento de Anatomia Patológica do Centro de Tratamento e Pesquisa do Hospital do Câncer A. C. Camargo Rua Prof. Antônio Prudente 109 - Liberdade CEP 01509-010 - São Paulo-SP

Tel.: (11) 3272-5103

Fax: (11) 3272-0495

e-mail: mdbegnami@hcancer.org.br 